\title{
Application of an Automated Surveillance System to Evaluate Outpatient Care of Pneumonia
}

\author{
Jeneen Gifford ${ }^{\star 1,2}$ and Sylvain Delisle ${ }^{1,2}$ \\ ${ }^{1}$ University of Maryland School of Medicine, Baltimore, MD, USA; ${ }^{2}$ Veterans Affairs Maryland Health Care System, Baltimore, MD, \\ USA
}

\section{Introduction}

With economic pressures to shift the care of community-acquired pneumonia (CAP) to the ambulatory setting, there is a need to ensure safety of outpatients with CAP. The use of claims data alone remains the primary strategy for identifying these patients, but billing information often does not match the clinical diagnosis and does not have the ability to find unrecognized cases. In our previous work, an automated pneumonia case detection algorithm (CDA) was able to detect cases of CAP with positive predictive value of $71 \% .{ }^{1}$ For this study, we begin to illustrate how this type of surveillance system may assist in evaluating the quality of outpatient care for CAP.

\section{Methods}

In this single-center, retrospective observational study, the pneumonia CDA was applied to electronic health record (EHR) documentation of all emergency room and outpatient clinic visits at the Baltimore VA Medical Center from 01/01/2004 to 12/31/2011. The pneumonia CDA consists of: 1) a CDA for acute respiratory infection (ARI) that uses ICD-9 codes, free text analysis of EHR notes to find symptoms, and records of cough remedy prescriptions to detect ARI cases with a sensitivity of $99 \%$, and 2) an automated chest imaging classifier that labels reports as non-negative (could possibly support a diagnosis of pneumonia) or negative. ${ }^{1} \mathrm{~A}$ manual chart review was completed for all visits flagged by the pneumonia CDA (detected by ARI CDA and having an associated non-negative chest imaging report). Exclusion criteria included non-initial visit, lack of new symptoms, and inpatient admission. "Nondismissable" pneumonia was defined as having [ 2 or more pneumonia symptoms AND a nonnegative chest imaging report AND (new or increasing chest imaging findings OR no prior chest imaging). ${ }^{2}$ Treatment was considered "timely" if prescribed prior to discharge from the emergency room or on the same date of the outpatient clinic visit. "Pneumonia in plan" describes the documentation of pneumonia as a possible diagnosis by the prescribing provider. For all visits, provider diagnosis, timeliness of treatment, and antibiotic choice were assessed.

\section{Results}

The pneumonia CDA flagged 666 visits over the 8-year study period. Of the 376 included cases, 271 (71.9\%) were found to be nondismissable. Providers missed pneumonia as a possible diagnosis in $95(35 \%)$ of 271 nondismissable cases. Nondismissable cases with pneumonia in plan $(n=176)$ received antibiotics, but in two cases, treatment was not timely. Thirty $(11.1 \%)$ of the Nondismissable cases had no pneumonia in plan and did not receive timely treatment, while 65 nondismissable cases (24\%) had no pneumonia in plan but received antibiotics for non-pneumonia ARI diagnoses. Fluoroquinolones were the most commonly prescribed antibiotic for pneumonia in plan (122 or $61.6 \%$ of 198) and for all visits (149 or $61.6 \%$ of 376 ). Macrolide monotherapy and doxycycline, which may be used to treat CAP in healthy patients with no risk for drug-resistant infection, ${ }^{2}$ were prescribed in $51(25.8 \%)$ and $12(6.1 \%)$, respectively, of visits with pneumonia in plan. The CDA also discovered cases where the provider suspected pneumonia, but prescribed trimethoprim/ sulfamethoxazole $(n=1)$ or beta-lactam monotherapy $(n=4)$ despite current treatment guidelines.

\section{Conclusions}

An automated CDA is a novel method to identify outpatient cases of CAP and can possibly uncover inadequacies in treatment. Future CAP studies may use CDAs to evaluate guideline adherence, treatment timing, both short-term and long-term outcomes, and overall quality of care.

Antibiotics Prescribed For Visits Flagged By the Pneumonia CDA

\begin{tabular}{|c|c|c|}
\hline Antibiotic prescribed (timely only) & Pneumonia in plan, $\mathbf{N}(\%)$ & All included visits, $\mathbf{N}(\%)$ \\
\hline Respiratory fluoroquinolone & $122(61.6)$ & $149(61.6)$ \\
\hline Beta lactam + macrolide & $1(0.5)$ & $1(0.3)$ \\
\hline Macrolide monotherapy & $51(25.8)$ & $75(19.9)$ \\
\hline Doxycycline & $12(6.1)$ & $38(10.1)$ \\
\hline Beta lactam monotherapy & $4(2.0)$ & $20(5.3)$ \\
\hline Trimethoprim/ sulfamethoxazole & $1(0.5)$ & $16(4.3)$ \\
\hline Other or not specified & $5(2.5)$ & $10(2.7)$ \\
\hline None & $2(1.0)$ & $67(17.8)$ \\
\hline Total & $198(100)$ & $376(100)$ \\
\hline
\end{tabular}

\section{Keywords}

Community-acquired pneumonia; Quality of care; Case detection; Surveillance

\section{References}

1. DeLisle S, Kim B, Deepak J, et al. Using the electronic medical record to identify community acquired pneumonia: toward a replicable automated strateg. PLoS ONE 2013;8;e70944.

2. Mandell LA, Wunderink RG, Anzueto A, et al. Infectious Diseases Society of America/American Thoracic Society consensus guidelines on the management of community-acquired pneumonia in adults. Clin Infect Dis 2007;44 Suppl 2:S27-72.
*Jeneen Gifford
E-mail: jgifford1@medicine.umaryland.edu 